as the lowest values for $\mathrm{FEV}_{1}$ that should be accepted for surgery without measurement of arterial blood gases.

Patients thus selected will benefit from preoperative physiotherapy and antibiotics, ${ }^{10}$ but we do not advocate any one anaesthetic technique as being superior to others for these patients. The important points are preoperative assessment and an awareness of the relevant pathophysiology. Spontaneous respiration seems acceptable for minor procedures not requiring relaxation; controlled ventilation is probably indicated in more prolonged anaesthesia. Regional analgesia for postoperative pain avoids the need for using morphine in patients who may have reduced respiratory sensitivity to carbon dioxide.

We thank Miss Anne Barrett who carried out most of the lung function tests and blood gas estimations and our surgical and anaesthetic colleagues who were involved in the care of these patients.

\section{References}

1 Diament, M. L., and Palmer, K. N. V., Lancet, 1967, 1, 1251.

2 Stein, M., et al., fournal of the American Medical Association, 1962, 181, 765 .

${ }^{3}$ Thornton, J. A., Problem of Anaesthesia and Surgery in Relation to Chronic Respiratory Disease, M.D. Thesis, University of London, 1969.

${ }^{4}$ Cotes, J. E., Lung Function, Assessment and Application in Medicine. Oxford, Blackwell, 1965.

${ }^{5}$ Raine, J. M., and Bishop, J. M., Fournal of Applied Physiology, 1963, 18, 284.

- Howard, P., British Medical fournal, 1972, 2, 89.

${ }^{7}$ Appleberg, M., Gordon, L., and Fatti, L. P., British fournal of Surgery, $1974,61,57$.

${ }^{8}$ Lockwood, P., Respiration, 1973, 30, 105.

${ }^{9}$ Andersen, N. B., and Ghia, J., Fournal of Thoracic and Cardiovascular Surgery, 1970, 59, 474.

${ }^{10}$ Stein, M., and Cassara, E. L., fournal of the American Medical Association, 1970, 211, 787.

\title{
Assessment of Regional Ventilation by Continuous Inhalation of Radioactive Krypton-81m
}

\author{
F. FAZIO, T. JONES
}

British Medical fournal, 1975, 3, 673-676

\section{Summary}

A simple technique is described for producing highquality functional images of regional ventilation during physiological tidal breathing of the inert gas ${ }^{81 \mathrm{~m}} \mathbf{K r}$. These images are quickly obtained on a gamma-camera without the need of computerized systems for data acquisition and display and are directly comparable with those of perfusion obtained with ${ }^{9} \mathrm{~mm}$ Tc-labelled macroaggregates. The short time required for the procedure, its simplicity, and the extremely low absorbed-radiation dose enable serial images of ventilation to be obtained in multiple views.

\section{Introduction}

There is a need for a technique to obtain functional images of ventilation that are comparable to the routinely obtained macroaggregate perfusion scan.' Previous methods have involved wash-in, wash-out, or a single breath of radioactive gases or aerosols of labelled particles. Because these methods tend to be either complex or inaccurate and unphysiological they have had limited clinical application.

We present here a new simple technique to obtain functional images of the regional ventilation using a gamma-camera during tidal breathing of the inert gas ${ }^{81 \mathrm{~m}} \mathrm{Kr}$. This method overcomes many of the limitations of previous techniques and when used in conjunction with perfusion data provides unique information on regional mismatching of ventilation and perfusion.

Department of Medicine, Hammersmith Hospital, London W12 0HS F. FAZIO, M.D., Wellcome Trust Research Fellow

M.R.C. Cyclotron Unit and Department of Diagnostic Radiology, Hammersmith Hospital, London, W12 0HS

T. JONES, M.sc., B.Sc., Medical Physicist

\section{Theory}

Continuous inhalation of an inert gas results in equilibration of the gas in the lungs. If a radioactive gas with a long half life is used the count rate at equilibrium, as measured externally over the chest, will be proportional to lung volume rather than to ventilation. Regional ventilation can be assessed only from the rate of equilibration (washin) or elimination (wash-out) of the gas.

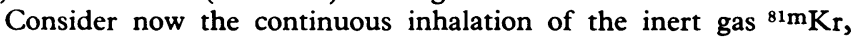
which has a radioactive half life of 13 seconds. The rapid radioactive decay of this gas relative to the ventilation turnover rate per unit volume will result in an alveolar concentration at equilibrium much less than that in the inspired air. Hence the contribution of the ${ }^{8} 1 \mathrm{mKr}$ wash-out to this dynamic process is small. Equilibration of the isotope in the lung therefore depends on the balance between arrival of ${ }^{81} \mathrm{mKr}$ and radioactive decay. ${ }^{81 \mathrm{~m} K r}$ lung counts are theoretically proportional to

$$
\frac{\dot{\mathrm{V}}}{\dot{\mathrm{V}} / \mathrm{vol}+\lambda}
$$

where $\dot{\mathrm{V}}$ is total ventilation, $\dot{\mathrm{V}} /$ vol the ventilation per unit of lung volume, and $\lambda$ the radioactive decay constant of $81 \mathrm{mKr}$, which is $3 \cdot 2 / \mathrm{min}$. The denominator is dominated by the high value for $\lambda$, hence the lung signal is more dependent on ventilation $(\dot{V})$ than on ventilatory wash-out $(\dot{\mathrm{V}} / \mathrm{vol})$. This results in an almost linear relation between radioactivity and regional ventilation for both normal (about $1.01 \mathrm{~min}^{-1} \mathrm{l}^{-1}$ ) and reduced ventilation rates in adults (fig. 1).

Therefore, the equilibrium images recorded with the gammacamera during the continuous inhalation of $81 \mathrm{~m} \mathrm{Kr}$ reflect the regional arrival of the gas-that is, ventilation. In other words, continuous tidal breathing of ${ }^{81 \mathrm{mKr}}$ effectively produces the same information as the sum of a series of separate tidal breaths of a radioactive gas with a long half life.

\section{Methods}

The ${ }^{81 \mathrm{~m} K r}$, which emits $190 \mathrm{keV}$ gamma-rays, can be produced continuously by passing air through a cation exchange column to which its parent isotope ${ }^{81} \mathrm{Rb}$ is bound. ${ }^{81} \mathrm{Rb}$ has a half life of four and a half hours and is produced for us on the M.R.C. cyclotron. ${ }^{2}$ The half life of the parent enables ${ }^{81 \mathrm{~m}} \mathrm{Kr}$ to be continuously available all day for ventilation measurements after a morning production of ${ }^{81} \mathrm{Rb}$.

If both ventilation and perfusion scans are required $1 \mathrm{mCi}$ of $99 \mathrm{mTc}$-labelled macroaggregates or microspheres (99m Tc-HAM) is first injected intravenously with the patient in the seated position. The patient is then positioned in front of the gamma-camera (Toshiba 


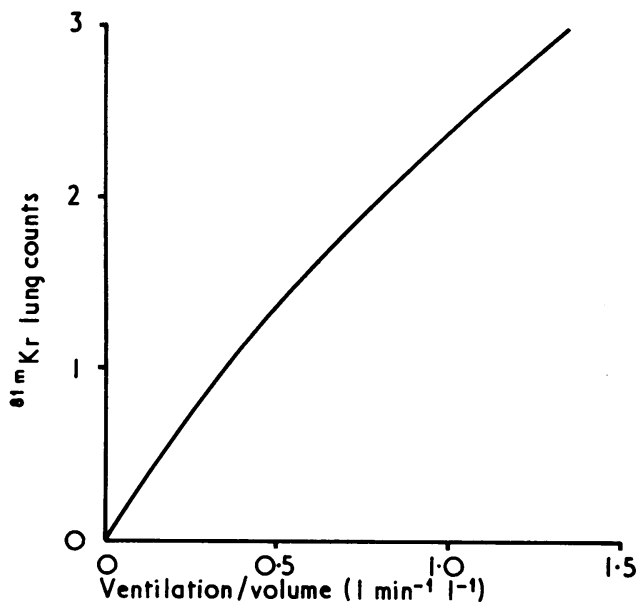

FIG. 1.-Theoretical relation between ${ }^{81 \mathrm{~m}} \mathrm{Kr}$ counts in lungs per unit of lung volume (see text).

Jumbo) and 300000 counts are collected with the window of the

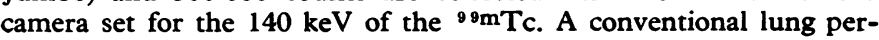
fusion image is recorded in this way on Polaroid or $35-\mathrm{mm}$ film. The patient is then asked to keep the same position and breathe normally through a disposable face mask while a constant supply of ${ }^{81 \mathrm{~m} K \mathrm{r}}$ continuously eluted from $10 \mathrm{mCi}$ of ${ }^{81} \mathrm{Rb}$ is added to the inspired air. The energy window of the camera is set for the $190 \mathrm{keV}$ gamma-rays of $81 \mathrm{mKr}$, and when, after some 30 seconds, equilibrium is reached ventilation pictures are taken, collecting again 300000 counts in about one minute. Spillover of $99 \mathrm{mTc}$ into the ${ }^{81 \mathrm{mKr}}$ window is less than $3 \%$. Both perfusion and ventilation images can be obtained in two to four minutes for each view. The total time required for a full study including anterior, posterior, and two lateral views is less than half an hour. The radiation-absorbed dose to the lungs for a ${ }^{81 \mathrm{~m}} \mathrm{Kr}$ study is $9 \mathrm{mrads}$ per view, which is about one-tenth of the dose of a standard $x$-ray examination of the chest. No waste disposal is required owing to the short half life of the isotope and its low concentration in the expired air.

\section{Results}

Fig. 2 shows a ${ }^{81 \mathrm{~m} K r}$ scan in an erect normal subject, showing the normal ventilation gradient. No day-to-day variations were observed in a group of 10 normal non-smokers studied repeatedly at various time intervals. Recently combined ventilation and perfusion scans have been performed at Hammersmith Hospital on 110 patients. Three examples of the method in patients with abnormal lung function are presented below.

Case 1. This 59-year-old man had chronic airways obstruction without radiological evidence of emphysema. We found gross defects of ventilation in both lungs (fig. 3 a). The defects were partially reversed after conventional treatment with antibiotics and $24 \%$ oxygen and after he stopped smoking. Fig. $3 \mathrm{~b}$ shows the ventilation image obtained in this patient after two weeks treatment. A much more uniform distribution of the ventilation is evident. The improvement of the scintigraphic patterns was accompanied by a

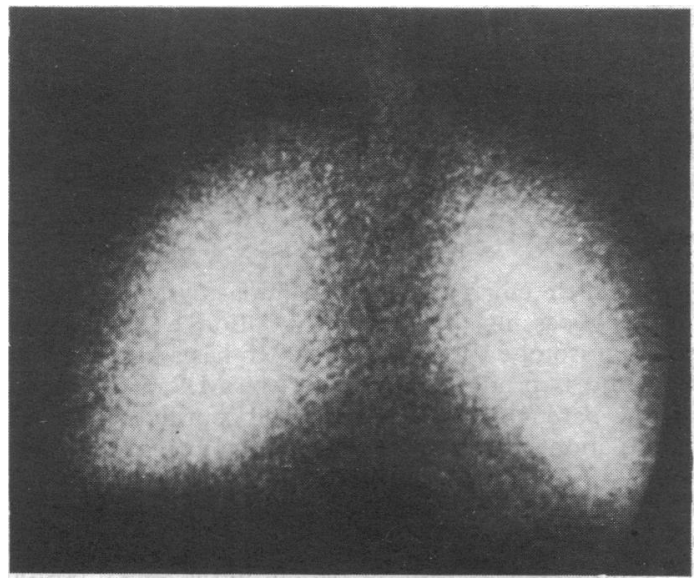

FIG. 2.-Ventilation image in normal subject (anterior view).

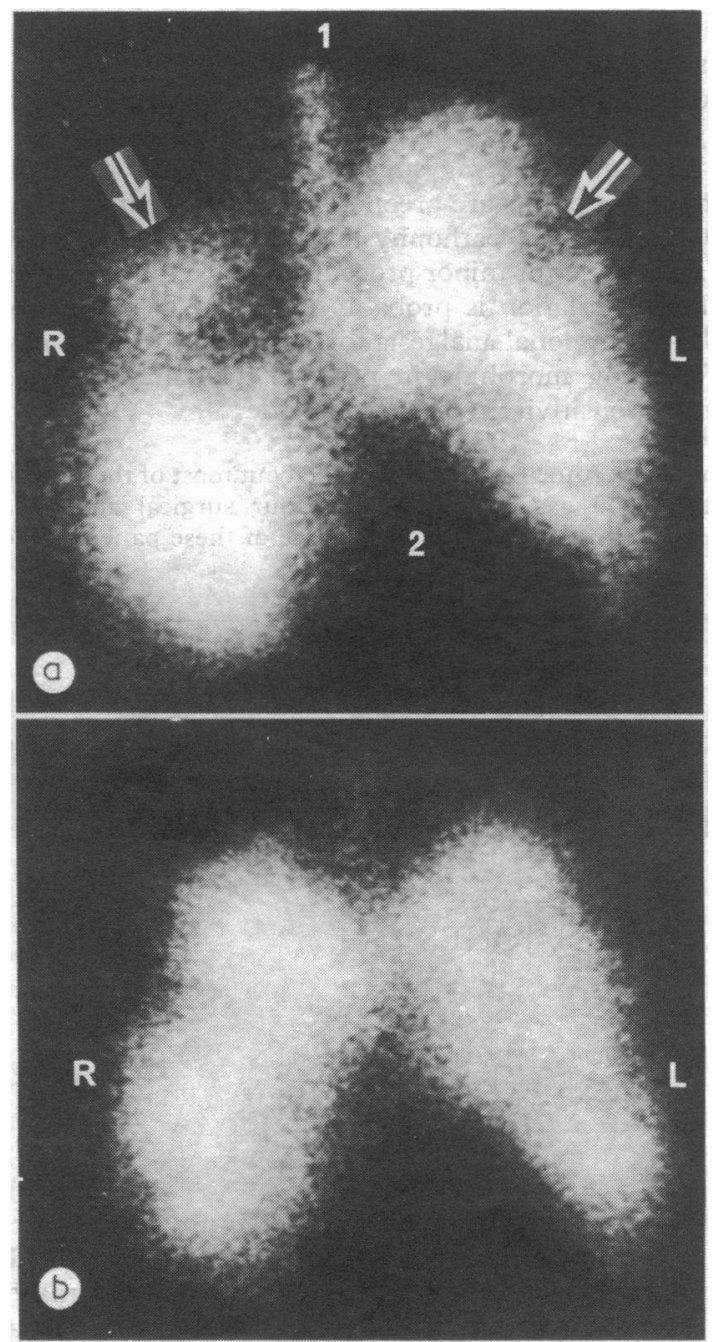

FIG. 3. $-{ }^{81} \mathrm{~m} \mathrm{Kr}$ ventilation scan (anterior view) in patient with chronic airways obstruction (a) before treatment showing gross defects of ventilation in both lungs (arrows). Ventilation is almost absent in upper part of right lung ( 1 =trachea; 2 =heart region); (b) after two weeks of treatment showing definite improvement in inhomogeneities of ventilation.

change in the lung function test results (vital capacity 1.4-3.2 l) and a significant reduction of the mixed venous carbon dioxide pressure $(10 \cdot 1-8 \cdot 0$ $\mathrm{kPa}(76-60 \mathrm{~mm} \mathrm{Hg})$ ).

Case 2.-This 55-year-old man had a history of myocardial infarction and recent onset of severe dyspnoea. The chest $x$-ray film showed an enlarged heart with clear lung fields. The lung perfusion scan in the anterior view showed a reduced perfusion in the lower part of the right lung, whereas the ${ }^{81 \mathrm{mKr}} \mathrm{Kentilation}$ scan was normal. Right lateral view showed a segmental defect of perfusion (fig. 4 a) in the presence of a normal ventilation (fig. 4 b). These findings were strongly suggestive of pulmonary embolism. ${ }^{34}$

Case 3.- To assess the validity of the method a comparison was made between the ${ }^{81} \mathrm{mKr}$ method and a reference technique- ${ }^{13} \mathrm{~N}$ wash-out-in one patient. A functional image of regional ventilation can be obtained by displaying, with the aid of a digital computer, the regional distribution of the mean wash=out rate of ${ }^{13} \mathrm{~N} .{ }^{5}$ Using ${ }^{81} \mathrm{mKr}$ in a patient with chronic bronchitis a defect of ventilation between the inferior and the middle third of the right lung was observed. A similar ventilatory defect was shown on the ${ }^{13} \mathrm{~N}$ functional image. Fig. 5 shows the intensity profile along a vertical strip through the right lung functional images for both ${ }^{81} \mathrm{mKr}$ and ${ }^{13} \mathrm{~N}$. The region of reduced ventilation was shown with both techniques. A sharper definition of this defect was obtained with ${ }^{81} \mathrm{mKr}$ than with ${ }^{13} \mathrm{~N}$ because of the higher spatial resolution obtained from the Anger gamma-camera when detecting the $190 \mathrm{keV}$ of ${ }^{81 \mathrm{mKr}}$ gamma-ray rather than the $511 \mathrm{keV}$ gamma-ray emitted by ${ }^{13} \mathrm{~N}$. Additionally, the extensive data manipulations necessary to obtain an image of the wash-out of ${ }^{13} \mathrm{~N}$ introduced a further loss in resolution.

\section{Discussion}

On theoretical grounds this new technique is valid below and within the normal range for tidal ventilation (see fig. 1). When 

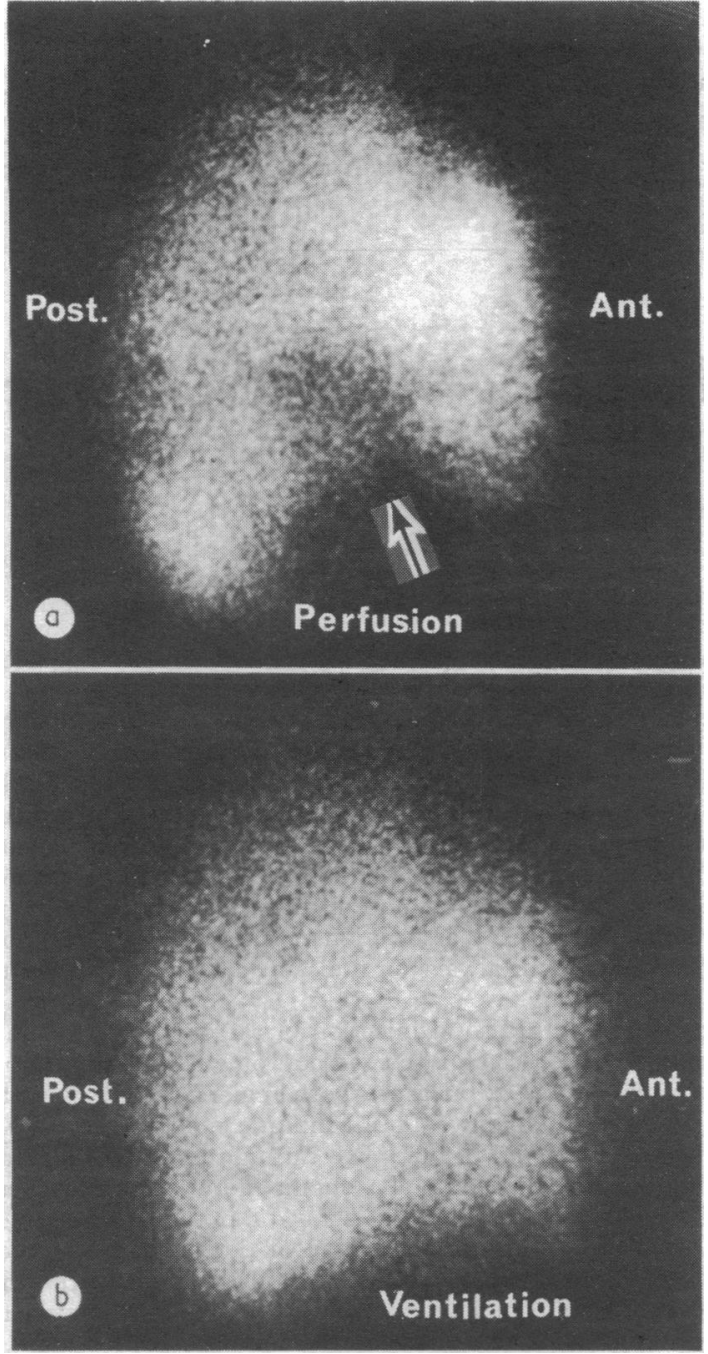

FIG. 4.-Perfusion and ventilation scans in patient with acute pulmonary embolism (right lateral view). (a) $99 \mathrm{mTc}$ microspheres perfusion scan showing segmental defect of perfusion (arrow). (b) ${ }^{* 1} \mathrm{~m} \mathrm{Kr}$ ventilation scan is normal.

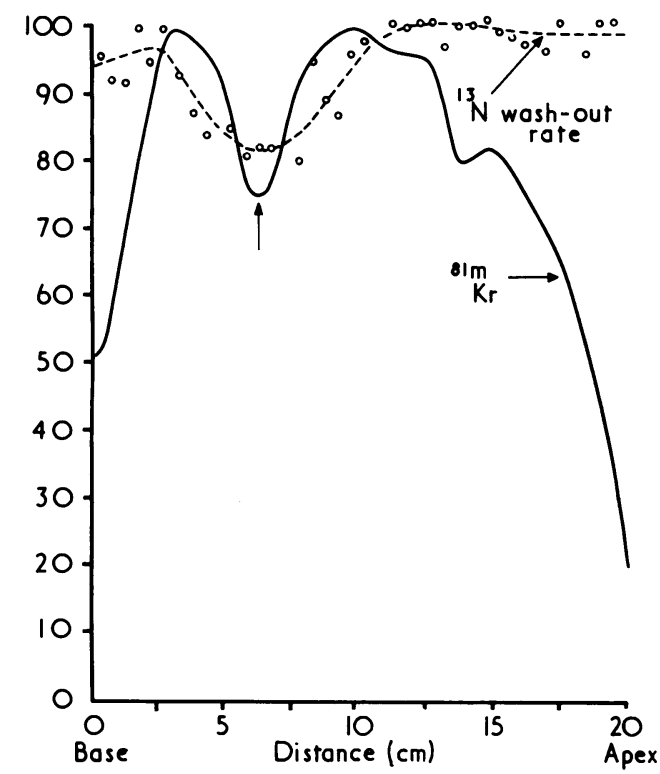

FIG. 5.-Distribution of ventilation in arbitrary units (ordinate) in vertical segment of right lung (abscissa) for patient with chronic bronchitis. ${ }^{81 \mathrm{~m}} \mathrm{Kr}$ measures total regional ventilation whereas ${ }^{13} \mathrm{~N}$ wash-out reflects ventilation per unit of lung volume, which accounts for general difference in shape. Region of reduced ventilation (arrow) is shown in both tracings but is more sharply resolved with $81 \mathrm{mKr}$. minute ventilation is greater than normal the signal tends to become dependent more on regional lung volume than on ventilation. But this is not a serious limitation for clinical assessment of poorly ventilated areas because they will equilibrate slowly even when total ventilation at the mouth is high.

There have been several different approaches to the study of regional ventilation with radioisotope imaging devices. ${ }^{4-9}$ Techniques involving wash-in or wash-out of "insoluble" radioactive inert gases such as ${ }^{133} \mathrm{Xe}$ or ${ }^{13} \mathrm{~N}$ have been used extensively. The significant solubility of ${ }^{133} \mathrm{Xe}^{11}$ introduces gross errors owing to uptake of tracer in the blood and in the chest wall that require appreciable corrections. ${ }^{8}$ Also, its low gammaray energy $(80 \mathrm{keV})$ prevents direct comparison with lung perfusion images obtained with ${ }^{99 \mathrm{~m} T \mathrm{~T}-H A M}(140 \mathrm{keV})$. If the highly insoluble gas ${ }^{13} \mathrm{~N}$ (half life 10 minutes) is used the solubility problems associated with xenon can be overcome. But ${ }^{13} \mathrm{~N}$ demands a cyclotron on line. Detection of the $511 \mathrm{keV}$ gamma-ray energy emitted by this isotope also results in poor sensitivity and spatial resolution with the Anger camera and is not comparable with that of ${ }^{99 \mathrm{~m}} \mathrm{Tc}$. With these techniques functional images can only be obtained with a computerized system for recording and analysing the data. Such procedures require a relatively long measurement time ( 20 minutes) and demand considerable expertise and technical resources. For these reasons these methods have not been used extensively in clinical practice.

Single-breath techniques using inert gases for imaging regional ventilation usually require a maximum inspiration and breath-holding manoeuvres. These are unphysiological and not representative of tidal ventilation. They usually result in poor-quality images owing to low counting statistics and are impracticable in dyspnoeic patients.

Areas of inhomogeneous ventilation can be shown in patients using labelled aerosol techniques, ${ }^{10}$ but measurements of regional ventilation with these methods have yet to be validated since they principally measure deposition of particles. Furthermore, this approach does not allow repeated measurements at short intervals because of the slow elimination of the activity from the lung.

With the ${ }^{81 \mathrm{~m}} \mathrm{Kr}$ continuous inhalation technique functional images of ventilation in multiple views can be obtained simply and quickly on a gamma-camera without the need of a computerized data acquisition and display system. These images are obtained during physiological tidal breathing and can be directly compared to images of regional lung perfusion obtained with ${ }^{99 m}$ Tc-HAM, because of the similar energy of the gamma-rays emitted from ${ }^{81 \mathrm{~m}} \mathrm{Kr}$ and ${ }^{99} \mathrm{~m} \mathrm{Tc}$. The source of ${ }^{81 \mathrm{~m} K r}$ is ${ }^{81} \mathrm{Rb}$, which is efficiently produced with a cyclotron 21213 and has a half life of four and a half hours. ${ }^{81 \mathrm{~m}} \mathrm{Kr}$ is therefore continuously available for up to 15 hours from the time of production of ${ }^{81} \mathrm{Rb}$. This makes the technique suitable for use in centres without a cyclotron.

This technique combined with the perfusion technique provides unique information for assessment of ventilation and perfusion mismatching in patients with lung diseases. We use it for diagnosing and managing pulmonary embolism, asthma, and chronic bronchitis and for the preoperative assessment of patients with emphysema, bronchiectasis, or pulmonary carcinoma.

We thank Professor C. Giuntini for stimulating discussion, Dr. J. M. B. Hughes and Dr. J. P. Lavender for encouragement and collaboration, Mr. J. C. Clark and Mr. T. A. Pratt for their technical expertise, and Mrs. Moira Stuart for typing the article.

F. Fazio is on leave from C.N.R. Laboratory of Clinical Physiology, Pisa, Italy, and this work has been supported in part by grants of the National Research Council of Italy (C.N.R.) and the British Heart Foundation.

\section{References}

1 Taplin, G. V., et al., fournal of Nuclear M॰dicine, 1964, 5, 259.

2 Jones, T., et al., Fournal of Nuclear Medicine, 1970, 11, 118. 
3 Wagner, H. N., et al., Radiology, 1968, 91, 1168.

4 DeNardo, G. L., et al., New England Fournal of Medicine, 1970, 282, 1334.

5 Nosil, J., et al., Physics in Medicine and Biology, in press.

- Newhouse, M. T., et al., Respiration Physiology, 1968, 4, 141.

7 Loken, M. K., and Westgate, H. D., Fournal of Nuclear Medicine, 1968, 9, 45.

${ }^{8}$ Secker-Walker, R. H., et al., fournal of Nuclear Medicine, 1973, 14, 725.
${ }^{2}$ Isawa, T., Hayes, M., and Taplin, G. V., fournal of Nuclear Medicine, $1971,12,606$

10 Santolicandro, A., and Giuntini, C., to be published.

11 Matthews, C. M. E., and Dollery, C. T., Clinical Science, 1965, 28, 573.

12 Yano, Y., McRae, J., and Anger, H. O., Fournal of Nuclear Medicine, 1970, $11,674$.

13 Colombetti, L. G., et al., fournal of Nuclear Medicine, 1974, 15, 868.

\title{
Changes in Cerebral Blood Flow during a Migraine Attack
}

\author{
J. W. NORRIS, V. C. HACHINSKI， P. W. COOPER
}

British Medical fournal, 1975, 3, 676-684

\section{Summary}

Regional cerebral blood flow studies during a typical prodromal phase of a migraine attack in a young woman showed a global decrease of cerebral blood flow in the carotid artery territory. These studies were repeated during the subsequent headache phase of the same attack and hemispheric blood flow increased considerably. Ergotamine tartrate was then administered intramuscularly which brought definite relief of symptoms but no change in cerebral blood flow. Carotid angiography performed immediately afterwards showed retrograde filling of the proximal portion of the basilar artery, which suggested that the brain stem was the site of hyperperfusion. These findings illustrate certain features underlying both the pathophysiology of migraine itself and its response to ergotamine preparations.

\section{Introduction}

It has been known for many years that migraine headache is associated with changes in the extracranial circulation, while the preheadache or prodromal phase is believed to be due to cerebral vasoconstriction. The observations of Wolff et al. ${ }^{1}$ largely confirmed this apparently paradoxical dissociation between extracranial and intracranial circulation, and, while the therapeutic effect of ergotamine compounds on the extracranial vasculature seems to be established, the response of the cerebral vessels remains unclear.

We recently performed serial estimations of cerebral blood flow (C.B.F.) in a young woman who fortuitously developed a migraine attack before angiography, and the resulting observations throw some light on the pathophysiology of this common though still ill-understood disorder.

\section{Case Report}

An 18-year-old unmarried woman had suffered typical migrainous left-sided hemicrania for two years before admission. The headaches were usually preceded by numbness in the right hand, which spread

MacLachlan Stroke Unit, Department of Neurosciences and Department of Radiology, Sunnybrook Medical Centre, Toronto, Ontario, Canada

J. W. NORRIS, M.D., M.R.C.P., Assistant Professor, Department of Neurosciences

V. C. HACHINSKI, M.D., F.R.C.P.(c.), Lecturer in Neurology

P. W. COOPER, M.D., F.R.C.P.(C.), Assistant Professor, Department of Radiology over the whole right side of the body, including the face, over about 10 minutes. This preheadache phase was often accompanied by a flickering right-sided hemianopia, which started as a tiny spot in the right field of vision and gradually extended over the full half of the visual field. A throbbing left-sided headache then developed, associated with occasional nausea and vomiting, and persisted for several hours. The prodromal phase disappeared rapidly as the headache phase became established. Routine analgesics were normally sufficient to abort the headache and she had never used ergotamine compounds. Because of recent episodes of unduly persistent hemianopia and the alleged, though infrequent, association of underlying arterial anomalies where symptoms occur always on the same side she was admitted for cerebral angiography.

Method. - The intracarotid injection of radioactive ${ }^{133} \mathrm{Xe}$ was used according to the well-established method of Lassen ${ }^{2}$ using a bank of 16 extracranial probes to measure both regional and global (hemispheric) cerebral blood flow. The technique modified for ${ }^{133} \mathrm{Xe}$ was used, ${ }^{3}$ and we injected $2-3 \mu \mathrm{Ci}{ }^{133} \mathrm{Xe}$ through an indwelling catheter in the internal carotid artery. To obtain more regional values than those offered by a strictly lateral array of probes we modified the probe holder to arrange a radial array of detectors. Both stochastic and compartmental values established in our laboratory are similar to those previously published, ${ }^{3}$ all the values for this patient being derived from the initial slope of the clearance curve (C.B.F. initial). Mean arterial blood pressure was continuously monitored via the intracarotid catheter, and blood gas analyses were determined by the Astrup technique performed on blood samples taken by the same route.

Procedure.-During the catheterization of the left internal carotid artery the patient began complaining of her usual prodromal symptoms, with progressive right hemianopia and right-sided numbness. The first regional C.B.F. estimation was then performed. About 15 minutes later the patient developed throbbing left hemicrania and the regional C.B.F. was estimated again. During this time she had increasing malaise and the headache increased in severity. During this second investigation $0.5 \mathrm{mg}$ of intramuscular ergotamine was given. By about 20 minutes after the second procedure none of the previous prodromal symptoms remained, and regional C.B.F. was estimated a third time. The patient's headache had largely disappeared, though a slight left throbbing hemicrania remained. After this procedure left carotid angiography was carried out.

Results.-C.B.F. values during the first procedure (prodromal phase) were uniformly low $\left(37.6 \pm 3.9 \mathrm{ml} 100 \mathrm{~g}\right.$ brain $^{-1}$ min $\left.^{-1}\right)$ and showed no regional differences (fig. 1a). In the second procedure, when the typical throbbing hemicrania was developing, hemispheric blood flow increased considerably $\left(63.3 \pm 6.2 \mathrm{ml} 100 \mathrm{~g}^{-1}\right.$ $\min ^{-1}$ ), but again there were no significant differences in regional values (fig. 1b). The patient was hyperventilating slightly during this phase and arterial carbon dioxide pressure $\left(\mathrm{PaCO}_{2}\right)$ fell so that the flow corrected for the same $\mathrm{PaCO}_{2}$ as in the first estimation was considerably increased ${ }^{3}$ (corrected value at $\mathrm{PaCO}_{2}$ of $5.3 \mathrm{kPa}$ (40 $\left.\mathrm{mm} \mathrm{Hg})=81.0 \mathrm{ml} 100 \mathrm{~g}^{-1} \mathrm{~min}^{-1}\right)$.

Finally, cerebral blood flow values at the third estimation, when the headache had largely disappeared, remained high $(63.5 \pm 10.3 \mathrm{ml}$ $100 \mathrm{~g}^{-1} \mathrm{~min}^{-1}$; fig. 1c) and were even higher if corrected for the $\mathrm{PaCc}_{2}$ (corrected value at $\mathrm{PaCO}_{2}$ of $5.3 \mathrm{kPa}=91.8 \mathrm{ml} 100 \mathrm{~g}^{-1} \mathrm{~min}^{-1}$ ). These results are summarized in the table.

The angiogram recorded at the end of the attack showed retrograde filling of the basilar artery from the posterior communicating artery. The other vessels, including the middle cerebral circulation, were normal (fig. 2). 